\title{
Materialien zur Entstehungsgeschichte von "Publik"
}

\author{
B) Abri $\beta$ der Planungs- und Vorbereitungsarbeiten
}

In diesem Heft setzen wir den Abdruck der von Michael BornefeldEttmann bearbeiteten Beilage fort, die der Entstehungsgeschichte der katholischen Wochenzeitung „Publik“ gewidmet ist. Teil A der Materialsammlung, die Bibliographie der Pressestimmen zu „Publik“ aus den Jahren 1966 bis 1968, erschien in den Ausgaben 1:1968/4 bis 2:1969/3. Es folgt hier Teil B der Materialsammlung: In chronologischer, jedoch zunächst keineswegs lückenloser Folge werden veröffentlicht: Protokollauszüge von Sitzungen der Planungs- und Vorbereitungsgremien, Auszüge aus Diskussionsbeiträgen und Vorschlägen mitwirkender Personen, Entwürfen, Gutachten und Projektstudien, Referaten, Tagesordnungen und Teilnehmerlisten.

Die Zusammenstellung, die mosaikartig die Entstehungsgeschichte verdeutlichen soll, kann noch kein geschlossenes Bild liefern. Eine im eigentlichen Sinne historische Darstellung muß späteren Zusammenfassungen vorbehalten bleiben. CS bittet um Mithilfe bei Ergänzung des Materials.

\section{September 1965}

Fulda, Plenarsitzung der Deutschen Bischofskonferenz. Darin ein Beschluß zur KWZ (= Katholische Wochenzeitung, Kürzel für das Projekt.) Niedergelegt im Protokoll „Fulda 1965, Nr. 45“. Diese Angabe nach: "Wortlaut des Beschlusses der Deutschen Bischofskonferenz zur Gründung einer katholischen Wochenzeitung. Fulda, September 1967.“

\section{September 1965}

Broschüre von Dr. Hans Suttner (damals Referent für staatsbürgerliche Angelegenheiten im Zentralkomitee der deutschen Katholiken, später erster Verlagsdirektor von „Publik", † 1968) mit dem Titel "Katholische Presse in Deutschland. Statt noch einer Kritik: ein konkreter Vorschlag", Regensburg 1965, 69 S., Privatdruck in kleiner Auflage, numerierte Exemplare. - Hier Auszüge.
S. $8 \mathrm{f}$ : "Kirche und Publizistik":

"Sie brauchen an dieser Stelle nicht Angst $\mathrm{zu}$ haben, es folgt keine allgemeine Erklärung, etwa des Inhalts: Wir müssen in der heutigen Zeit ja sagen zu den modernen Mitteln... An Äußerungen dieser Art fehlt es nicht, es ist überflüssig, diesen Bekenntnissen ein weiteres hinzuzufügen.

Dennoch ist ein grundsätzliches Wort vonnöten. Dieses grundsätzliche Wort gilt dem Problem, ob und wie hier und heute ,das Katholische publizistisch repräsentiert werden kann. [...]

So richtig es ist, daß es nach wie vor katholische Presseorgane geben muß, die institutionell der kirchlichen Hierarchie ein- oder angegliedert sind, so verkehrt wäre es, die Möglichkeit katholischer Publizistik ohne diese institutionelle Bindung $\mathrm{zu}$ leugnen. Entgegen einer verbreiteten Meinung bleibt diese Publizistik auch dann ,katholisch', wenn sie sich nicht allein an den katholischen Leser 
wendet, sondern die Darstellung des Katholischen im Gespräch mit den anderen sein will. Die Gegenmeinung, die sich darauf beruft, ,niemand könne zwei Herren dienen', übersieht, daß Dienst am anderen nicht Preisgabe des Katholischen ist." [...]

S. $13 \mathrm{ff}$.:

"Der entscheidende Vorzug einer katholischen Redaktion ist der, daß ein gewisser Minimalbestand von Überzeugung nicht dauernd in Frage gestellt werden muß. Somit ist eine Integrationsbasis vorhanden, die ein $A$ u f b a u e n möglich macht. Auch der Leser weiß um diese Gemeinsamkeit, sei es, daß er die Zeitung deswegen - grundsätzlich als die seine ansieht, sei es, daß er mit katholischer Meinung ins Gespräch kommen will.

Anläßlich der Verlegerkonferenz der ,Arbeitsgemeinschaft Kirchliche Presse' wurde im April 1965 folgender Satz geprägt: ,Die evangelischen Verhältnisse lassen eine solche Wochenzeitung [gemeint: ,Christ und Welt'] $\mathrm{zu}$, im katholischen Raum liegt eine so breite und ganz allein der Redaktion überlassene Meinungsbildung gar nicht im Bereich des Möglichen.' Dieser Satz besagt nichts anderes, als daß katholische Presse als Laieninitiative nicht möglich ist. Er kann deswegen nicht richtig sein. [...]

,Katholische Presse' wurde oben unter anderem damit gekennzeichnet, daß die grundsätzlichen katholischen Wahrheiten nicht in Frage gestellt worden. Insoweit ist eine katholische Presse ohnehin automatisch an das Lehramt der Kirche gebunden. Verlangen, daß sich diese Bindung in der Anerkennung einer Zensur dokumentieren müßte, hieße Mißtrauen statt Vertrauen zur Basis machen. Andererseits darf katholische Presse nicht vor Kritik geschützt und nicht von Kritik verschont werden. Die Kritik an der Presse wird aber notwendigerweise eine ö $f f e n t$ liche (im Sinne von: öffentlich geäußerte) sein müssen. Die anderwärts gängigen Praktiken, jemanden ,hintenherum abzuschießen sollten (leider muß die Wunschform gebraucht werden) einer katholischen Presse, die sich der Wahrheit verpflichtet weiß, unwürdig sein.

Die Form, in der die Vertreter der Hierarchie in der katholischen Presse (kritisierend) das Wort ergreifen, muß also der Eigenart der Presse gerecht werden. Diese
Form wird außerdem variabel sein müssen, je nachdem, ob ein Vertreter der Hierarchie kraft seines Amtes sich äußert oder ob einer von einem Laien geäußerten Meinung eine andere persönliche Meinung entgegengesetzt werden soll.

Nicht nur in der oben erwähnten Äußerung eines katholischen Verlegers, auch anderwärts wird die Wandlung der katholischen Presse vom Sprachrohr der Hierarchie hin zum Forum katholischer Laien vorwiegend als $\mathrm{G}$ e $\mathrm{f} \mathrm{h} \mathrm{r}$ angesehen. Demgegenuiber sind auch die Vorteile eines solchen Wandels zu beachten: Die katholische Presse kann so endlich der Fehldeutung entrinnen, sie dürfe sich, wenn überhaupt, nur endgültig, definitiv äußern. Wird diese Presse nämlich zuerst als Laien-Forum betrachtet, so leuchtet ohne weiteres ein, daß zwar grundsätzlich fundierte, aber dennoch nicht auf institutionelle Autorität gegründete $\mathrm{Me}$ in ung kundgetan wird. Der katholische Journalist $\mathrm{X}$ kann in einer Weise, die er selbst vera ntwortet, prononcierter Stellung zu Zeiterscheinungen nehmen, als es etwa ein Bisch of kraft seines Amtes könnte. So wäre gewährleistet, daß die katholische Presse eine aktive Zelle der öffentlichen Meinungsbildung darstellt. Das eben Gesagte gilt für einige Gruppen von katholischen Presseorganen nicht. $\mathrm{Zu}$ nennen sind vor allem die Bistumszeitungen. Sofern sich diese Zeitungen (mit Recht?) als Seelsorgsorgan des Bischofs verstehen, liegen andere Gesetzmäßigkeiten zugrunde. Diese Presseorgane können dann nichts anderes sein als ein Sprachrohr der Hierarchie. Daß damit freilich eine thematische Einengung verbunden ist, liegt auf der Hand."

S. $17 \mathrm{f}$. "Das ,Journalistische' als eigentlicher Qualitätsmaßstab“:

„Viele Diskussionen um die Qualität der derzeitigen katholischen Presse scheitern, weil sich die Diskutierenden nicht darüber klar sind, welche Qualitätsmaßstäbe anzulegen sind. [...]

Klassische journalistische Forderung, demokratisches Verständnis und Lehre der Kirche treffen sich in dem für den Publizisten entscheidenden Punkt der Faktizität der vermittelten Information. Das, Recht auf Information' ist ja zunächst ein Recht darauf, mit den Tatsachen bekanntgemacht $\mathrm{zu}$ werden, die eine zuverlässige Meinungsbildung erst er- 
möglichen. Dem Recht entspricht auf der Seite des Publizierenden eine Pflicht zur Wahrheitsliebe und das heißt zunächst zur Fakten-Treue. [...] Mit dem Stichwort, Unabhängigkeit' ist ein zentrales Problem aller Gesinnungspresse berührt. Einerseits will diese Presse als solche einer bestimmten Gesinnung dienen, andererseits soll der einzelne Journalist seine Meinung kundtun. Berüdksichtigt man, daß schon andere $\mathrm{Ab}$ hängigkeiten oder Unabhängigkeitsansprüche den Beruf des Journalisten seit eh und je belasten (etwa solche, die mit dem Verhältnis Verlag - Redaktion zusammenhängen), so wird wohl ohne weiteres deutlich, daß Spannungen nahezu unvermeidlich sind.

Das Problem wird manchmal - so hat es für den unbefangenen Außenstehenden den Anschein - dadurch gelöst, daß die Unabhängigkeit des Journalisten zugunsten einer ins einzelne gehenden Weisungsgebundenheit verdrängt wird. Wo immer dies der Fall ist, kann keine journalistische Leistung gedeihen. Die Form der Auseinandersetzung mit dem Journalisten und die Form der Korrektur seiner Meinung ist die $\mathrm{Kritik}$, nicht die $Z$ ensur. Im übrigen gibt es genügend Beispiele (gerade aus der jüngsten Zeit) dafür, daß unbequeme Kritiker, die im eigenen Lager der katholischen Gesinnungspresse nicht zu Wort kommen konnten, sich hier also auch nicht der Kritik stellen konnten und mußten, in andere Bereiche der Publizistik abgewandert sind und dort eine Resonanz erlangt haben, die sie für katholische Kritik nahezu unerreichbar machte. [...] Zưm Zwecke sachgerechter Beurteilung müssen an die katholische Presse diese (und viele weitere) journalist $\mathrm{i}$ s ch e Maßstäbe angelegt werden." [...]

S. 22 ff. "Struktur der heutigen Presse; katholische Präsenz in den einzelnen strukturellen Bereichen ${ }^{\alpha}$ :

"Die heutige periodisch erscheinende Presse weist verschiedene mehr oder minder deutlich abgrenzbare Strukturtypen auf. Mit Begriffen wie ,überregionale Tageszeitung, ,Frauenzeitschrift', ,Illustrierte', ,Wochenmagazin', Wochenzeitung" verbindet jedermann eine bestimmte Vorstellung. Auch Menschen, die diese Vorstellung nicht definieren oder audh nur beschreiben können, haben sie. [...] Es ist unverantwortlich, durch eine nicht zeitgerechte Aufmachung dem Menschen die- ser Zeit, der ja mit ganz bestimmten Qualitätsvorstellungen ,nach einer Zeitung greift', inhaltlich gute und widhtige Außerungen vorzuenthalten. [...] Schon bei oberflächlicher Betrachtung, erst recht bei genauerer Untersuchung, zeigt sich, daß die katholischen Stimmen einige Strukturformen von Zeitschriften besonders bevorzugen, während anandere Formen überhaupt nicht als Medium gewählt werden. [...]

Die Bistumsblätter sind während der vergangenen Monate in den Mittelpunkt der Auseinandersetzung gerüdkt. So hat sidh z. B. die Diskussion um eine ,Konzentration ' der katholischen Presse vorwiegend mit den Diözesanblättern beschäftigt. Daß gerade den Bistumsblättern diese zentrale Stellung zuerkannt wird, ist kein Zufall. Diese Zeitschriftengruppe hat zusammen eine Auflage, die sie auf einen der vordersten Plätze der wöchentlich erscheinenden Periodika stellt. Diese Auflage legt den Gedanken nahe, aus den 22 Bistumsblättern, die große katholische Zeitung' zu machen. Der Vorschlag hat ebenso leidenschaftliche Befürworter (außerhalb der Redaktionen der Bistumsblätter) wie leidenschaftliche Gegner (vorwiegend in den Reihen der Redakteure und Verleger der Bistumsblätter). Leider sind die Fronten in dieser Auseinandersetzung so starr, daß eine sachgerechte Diskussion kaum zustandekommt. So kann es nicht ausbleiben, daß Affekte vor Argumenten rangieren. Obwohl also, wie gesagt, wenig Aussicht auf Gehör besteht, sollen einige Gesichtspunkte zu dieser Frage vorgetragen werden:

(1) Die Bistumsblätter können der ursprünglichen Idee entsprechend als Organ des Bischofs, als Forum der Diözesanfamilie begriffen werden. Ihre seelsorgliche un $\mathrm{d}$ regionale Beschränkung müßte dann in der thematischen Begrenzung sichtbar werden.

(2) Den Bistumsblättern sind (z. T. durch die Nachkriegsumstände) auch Aufgaben zugefallen, die an sich nicht in einem notwendigen Zusammenhang mit der unter (1) geschilderten Funktion stehen (Unterhaltung, Politik). Diese Aufgaben können heute, nachdem völlige Pressefreiheit wiederhergestellt ist, auch von anderen Organen wahrgenommen werden.

(3) Mit dem Argument (1) kann die diözesane Eigenständigkeit der Bistumsblätter gerechtfertigt werden, für die Aufgaben, die 
den Bistumsblättern nebenher zugefallen sind, kann der Anspruch auf diözesane Eigenständigkeit nicht erhoben werden. Vor allem geht es nicht an, den sachlich gerechtfertigten Anspruch auf ein zweites Aufgabenfeld wahllos zu übertragen bzw. auszudehnen.

(4) Der oft ergänzend herangezogene Gesichtspunkt ,regionale Eigenart ist nicht sehr stichhaltig, $d_{a}$ es regionale Eigenart im Sinne der Eigenständigkeit einer B is t u m sbevölkerung kaum noch irgendwo gibt. Regionale Eigenarten sind heute jedenfalls weitergespannt, ganz abgesehen davon, daß regionale Eigenart gegenüber den durch die Berufswelt bedingten Unterschieden zurüdktritt.

(5) Die entscheidende Frage lautet wohl; will der Bezieher des Bistumsblatts neben dem Organ der diözesanen Seelsorge a u $\mathrm{ch}$ die (religiös fundierte) Wochenzeitung (vgl. oben (2)? Will der Bezieher diese Wochenzeitung nicht, mitgeliefert', so sollte man die Bistumsblätter von all diesen Elementen entlasten und statt dessen für katholische Wochenzeitungen werben. Will der Bezieher aber die Wochenzeitung mitgeliefert haben, so sollten alle Möglichkeiten geprüft werden, die einer konzentrierten systematischen Arbeit an einer solchen wirklich aktuellen Zeitung dienen. Daß eine solche Wochenzeitung von den Redaktionen der bisherigen Bistumsblätter nicht getrennt erarbeitet werden kann, liegt auf der Hand. Die Qualität einer Wochenzeitung ist heute nicht mehr nur eine Frage des guten Willens oder des per$\mathrm{s}$ ö $\mathrm{l}$ i c h e n Talents eines Redakteurs: Behaupten kann sich nur die Gemeinschaftsleistung."

S. $27 \mathrm{ff} .:$ „,Katholische Presse“ - ein Modell" :

"Wenn von der ,Katholischen Presse die Rede ist, so wird in aller Regel (vernünftigerweise) nach Vorhandenem gesehen. Das Ziel dieser Schrift ist es, in die Zukunft zu weisen, deswegen muß auch die Frage nach dem Möglichen, dem Nötigen und dennoch nicht Vorhandenen, aufgeworfen werden. Die Frage lautet konkret: ,Welche Presse brauhen wir?' Ohne Anspruch auf Vollständigkeit erheben $\mathrm{zu}$ wollen, nenne ich:

- Das Diözesanblatt als Organ des Bischofs.

- Die Wodhenzeitung als Mittlerin kultu- reller und grundsatzpolitischer Einsichten und Zusammenhänge.

- Die überregionale Tageszeitung als Ergänzung des Lokalblatts. Sie soll den politisch aktiven Teil der katholischen Bevölkerung ansprechen und permanent fortbilden.

- Wo möglich das Lokalblatt.

- Das wöchentlich erscheinende Nachrichtenmagazin höchstaktuellen, kritischen und polemischen Charakters.

- Die Illustrierte in technisch anspruchsvollster Aufmachung, mit spezifisch auf ein breites Publikum zugeschnittenem Inhalt.

- Die Fernseh- und Funk-Illustrierte herkömmlicher Art.

- Die Frauenzeitschrift anspruchsvoller (vom Blickpunkt der Durchschnittsfrau aus gesehen!) Aufmachung.

- Die Jugendillustrierte, die sich nicht in erster Linie an ,organisierte Jugend' wendet.

- Das Unterhaltungsblatt für die konser. vative, ländlich/bäuerliche oder bürgerlich/ traditionelle Bevölkerung.

- Das (Tages- oder Wochen-)Blatt im Boulevardstil für d e n Bevölkerungsteil, der von seiner Milieu-Prägung her unfähig ist, eine anders aufgemachte Gesinnungspresse zu lesen.

- Die betont kritische Monatszeitschrift für höhere Schüler und Studenten (evtl. zwei im Anspruch gesteigerte Ausgaben).

- Die Kinder-Illustrierte, die sittlide, religiöse und geschmadkliche Grundlagen in pädagogisch höchsten Anforderungen genügender Weise vermittelt. [...]

Eine solche Wunschliste ist natürlich ohne jeden Wert, wenn sie sich nicht in die Tat umsetzen läßt. [...]

Das Hauptproblem ist der personelle Mangel. $[\ldots]^{\alpha}$

Folgerichtig macht Suttner (S. $42 \mathrm{ff}$.) den konkreten Vorschlag zur Gründung einer "ausschließlich dienenden und helfenden" Institution, des - in seiner Schrift so genannten - „Instituts Publizistik" (IP). Dieses soll nach Suttner - hier in Kurzfassung - folgende Aufgaben wahrnehmen:

- Aufbau und Pflege eines funktionsfähigen Pressearchivs.

- Aufbau und Pflege einer Kartei freier Mitarbeiter. 
- Bildung eines schlagkräftigen Teams junger Publizisten.

- Schaffung und Betreuung einer Journalistenschule neuen Stils.

- Veranstaltung und Anregung von Fortbildungskursen.

- Anregung wissenschaftlicher Untersuchungen über die Lage und die Chancen katholischer Pressearbeit.
- Systematische Talentsuche für publizistische Berufe.

- Unterstützung aller Gemeinschaftsunternehmungen katholischer Presseorgane hinsichtlich Werbung und Vertrieb.

- Intensive Zusammenarbeit mit Redaktionen ausländischer Zeitschriften.

(Wird fortgesetzt.)

BEILAGE II

\section{Klaus Merten - Frogard Nölting - Michael Schmolke}

\section{Fachlich-biographische Notizen zur publizistischen Tätigkeit katholischer Geistlicher in der deutschen Presse (19. Jh.)}

Mit dieser 10. Folge schließen wir die Beilage ab. Eine allgemeine Einführung in die fachlich-biograph. Notizen findet der Leser in CS Nr. 1/1968, S. 74-76. $\mathrm{Zu}$ wiederholen ist der Hinweis, daß für die Auswahl der erwähnten Personen ihre Aktivitäten in der Publizistik, also vornehmlich in Zeitungen und Zeitschriften, wichtig waren. Die meisten Angaben wurden aus Sekundärquellen gewonnen. Auf die Belege wurde hier mit Chiffren hingewiesen, deren Auflösung auf Seite 208 $\mathrm{zu}$ finden ist. Hinweise und Korrekturen sind der Redaktion auch nach Abschluß der Serie noch willkommen. Für bereits erfolgte Ergänzungen sei an dieser Stelle gedankt.

\section{0. (und letzte) Folge}

Hemlinger, Stadtpfarrer in Trier, 1848 Gründer des Katholischen Volksboten für Stadt und Land. 1 II, 168.

Retzbach, Anton, Herausgeber und Chefredakteur, - 13. 6. 1867 Berolzheim in Baden, Dr. theol. et rer. pol., 1903 Dompräbendar, Leiter der katholischen Árbeitervereine der Diözese und 15 Jahre Chefredakteur der Soxlalen Revue, 1925 Badischer Landtagsabgeordneter. KK, 3919. - Mitherausgeber der Sozialen Revue. 9, 94.

Reusch, Franz Heinrich, Herausgeber, Chefredakteur und Mitarbeiter, '4. 12. 1825 Brilon, +3 . 3 . 1900 Bonn, 1849 Priester, 1858 Professor, 1872 exkommuniziert, seitdem altkatholischer Generalvikar. Herausgeber des Theologischen Literaturblattes 1866-77. KK, 3922. - Chefredakteur der Kölnischen Blätter. 9, 40, vgl. 14, 14. - HerausBeber des Bonner Theologischen Literaturblattes.
9, 45. - Mitarbeiter des Chlllaneums. 9, 44. Mitarbeiter der Deutschen Volkshalle seit 1850 , später auch Redakteur. 1 II, 238. - Mitarbeiter an der Deutschland. 1 II, 443.

Richter, Franz, Redakteur, * 9. 10. 1845 Osterwick, 1871 Priester, Dechant in Bocholt, Redakteur der Duisburger Volkszellung 1873-1880. 6, 50.

Rieder, Karl Joseph, Redakteur, * 9. 2.1876 Emmendingen in Baden, + 3./4. 9. 1931 ReichenauNiederzell, 1899 Priester, Pfarrer in ReichenauNiederzell, 1910-1919 Redakteur des Frelburger Diözesanarchivs. KK, 3933.

Riedinger, Friedrich Dominikus, Domkapitular in Speyer, Redakteur des Chrlstllchen Pilgers. 18, 231. 
Riegl, Anton, Mitarbeiter, * 12. 2, 1879 Salzburg, 1901 Priester, Religionslehrer, Mitarbeiter des Linzer Volksblattes. KK, 3965.

Ries, Josef, Chefredakteur und Mitarbeiter, * 9. 12. 1867 Boxberg in Baden, $t$ 19. 6. 1932 Freiburg, 1912 Regens des Freiburger Priesterseminars, Schriftleiter und Mitarbeiter des Oberrhelnischen Pastoralblattes. KK, 3973 .

Riess, S.J, Florian Joseph, Gründer, Chefredakteur und Mitarbeiter, ${ }^{\circ}$ 5. 2. 1823 Tiefenbach in Württemberg, t 30.12 .1882 Feldkirch, 1845 Priester, Gründer und Chefredakteur des Deutschen Volksblattes in Stuttgart, Mitbegründer der Stimmen aus Maria-Laach und deren Mitarbeiter, 1850 Gründer des Kalholischen Sonntagsblattes in Stuttgart, 1870 Professor für Kirchengeschichte. KK 3975. - 1848 Begründer und 1848-57 auch Redakteur des Deutschen Volksblattes in Stuttgart. 6,14 , vgl. 9,33 und 1 II, 163 .

Riffel, Kaspar, Gründer, Redakteur und Mitarbeiter, ${ }^{+19}$. 1. 1807 Budesheim in Rheinhessen, +15. 12. 1856 Mainz, 1830 Priester, 1835 Professor, 1841 Redakteur des Katholik, Mitbegründer des Piusvereins und des Mainzer Journals. KK, 3980 . 1842 Gründer der Katholischen Sonntagsblätter. 4, 128, vgl. 9, 24. - Mitarbeiter des Katholik. 13, 12, vgl. 4, 130. - Mitarbeiter der Katholisehen Sonntagsblätter zur Belehrung und Erbauung. 13, 217.

Ritter, Joseph Ignaz, IIerausgeber, *12.4. 1787 Schweinitz in Schlesien, + 5. 1. 1857 Breslau, 1811 Priesterweihe, 1823 Professor, 1840 Kapitularvikar und später Generalvikar in Breslau. KK, 4006. llerausgeber der Jahrbücher zur Verbreitung des Glaubens. 1 I, 252.

Rittler, Aloys, Gründer, Herausgeber und Chefredakteur, *26. 1. 1839 Jedesheim bei Illertissen, t 5. 8. 1890 Brennerbad in Tírol, 1865 Priester, 1871 Gründer der Periodischen Blätter in Regensburg, Chefredakteur des Fränkischen Volksblatles in Würzburg und Herausgeber der Katholischen Fahne in München, 1875 Mitglied des bayrischen Landtages, 1882 Professor für Philosophie. KK, 4010. - Seit 1877 Herausgeber der Katholischen Fahne. 19, 62. - 1869-72 Chefredakteur des Münchener Volksboten. 1 I, 282.

Rody, Heinrich, Pfarrer in Ostrich am Rhein, * 1805. 6, 25. - Redakteur der Katholischen Bewegung, 19,78 , vgl. 7 II, 307 und 6.25 .

Röcker, Kaplan, Schriftführer des Piusvereins, Redakteur des Pius IX., Christlich-Demokratische Wochenschrift. 1 II, 3.

Rolfus, Hermann, Chefredakteur, * 24. 5. 1821 Freiburg, † 27. 10. 1896 Bühl, 1844 Priester, Ehrendoktor in Freiburg und Geistlicher Rat, Chefredakteur des Süddeutschen Katholischen Schulwochenblattes. KK, 4037.

Rüsche, Franz, Herausgeber, * 29. 3. 1888 Bleche in Westfalen, Dr. theol. et phil., Professor in Paderborn, Mitherausgeber von Theologle und Glaube. KK, 4095.

Rütjes, Heinrich Gisbert, Mitarbeiter, * 4. 11. 1811 Emmerich $+20,2,1886$ ebenda, 1836 Priester, Dr. theol., 1847 Pfarrer in Obermörmter. KK, 4097. - Mitarbeiter des Rheinischen Kirchenblattes. 13, 54, vgl. 13, 219. - Mitarbeiter der Katholischen Blätter. 13, 221.

Ruland, J. N., Herausgeber, Redakteur und Mitarbeiter, Kaplan an St. Hedwig in Berlin, Mitbegründer des Bonifatius-Vereins. 13, 193 . 1847 Herausgeber und Redakteur des Petrus und Paulus in Berlin. 13, 226. - Mitarbeiter des Chilianeum. 9,44 .
Rump, Hermann Heinrich, Gründer und Redak teur, * 1. 3. 1830 Essen in Oldenburg, $t 21$. 22. 8. 1875 St. Mauritz bei Münster, 1853 Priester. gründete 1861 zusammen mit Hülskamp den Literarischen Handweiser. $K K, 4106$; vgl. 9, 19, 45, 124.

Rußwurm, Heinrich, Redakteur, * 5. 9.1802 Gremsdorf in Oberfranken, $t$ 23. 10. 1868 Landau an der Isar, 1827 Priester, Geistlicher Rat in Landau, Landtagsabgeordneter. KK, 4114. - 1841 bis 1845 Redakteur der Passauer Katholischen Kirchenzeitung. 13, 214.

Sägmüller, Johannes Baptist, Herausgeber, * 24 2. 1860 Winterreute bei Biberach, 1884 Priesterweibe, 1888 Prolessor in Tübingen, Mitherausgeber der Tübinger Theologisehen Quartalsehrift. KK, 4130.

Saffenreuther, Georg Joseph, Herausgeber, Chefredakteur, Redakteur und Mitarbeiter, - 8. 7 1808 Bamberg, t 17. 5. 1869 Würzburg, 1831 Priesterweihe, Ehrendoktor in Würzburg, Chef redakteur des Religions- und Kirchenfreunds (seit 1840 Allgemeiner Religions- und Kirchenkorrespondent). KK, 4131. - Seit 1841 Herausgeber und Redakteur, ab 1844 auch Chefredakteur des Religionsfreund für Katholiken. 13, 200. Seit 1840 Jerausgeber des Allgemeinen Religionsfreund ftir Katholiken. 1 I, 245. - Mitarbeiter des Chilianeum. 9,44 .

Sailer SJ, Johann Michael (von), Mitarbeiter, * 17. 11. 1751 Aresing bei Schrobenhausen in Oberbayern, $t 20$. 5. 1832 Regensburg, 1784 Professor für Moral- und Pastoraltheologie in Dillingen, 1829 Bisch of in Regensburg. KK, 4134. Mitarbeiter des Katholik. 13, 141. - Mitarbeiter des Religionsfreundes für Katholiken und der Literaturzeitschrift für katholische Religionsleh rer. 13, 143. - Mitarbeiter der Eos in München. 9, 25 .

Sartorius, Johannes, Herausgeber, * 26. 5. 1870 Mainz, 1896 Priester, Lehrer in Mainz, $1912 \mathrm{Her}$ ausgeber der Jugendzeitschrift Die Burg. KK, 4165 .

Sauer, Joseph, Gründer und Chefredakteur, 27. 11. 1803 Spurwitz in Schlesien, +24.6 .1868 Breslau, 1827 Priester, Professor, 1848 Domherr in Breslau, mit Thiel Gründer des Sehlesisehen Kirchenblattes 1835 und Chefredakteur bis 1848 . KK, 4171 . 13, 208, vgl. 1 I, 246, sowie 9,23 .

Sauer, Joseph, Herausgeber, * 7. 6. 1872 Unzhurst in Baden, 1898 Priester, 1905 Professor in Freiburg, Herausgeber der Literarischen Rundschau 1905-14 und des Kunst wissenschaftlichen Jahrbuches der Görresgesellsehaft, Landeskonservator in Baden. KK, 4171. - Herausgeber der Literarischen Rundschau. 9,93 .

Sauerborn, Kaplan in Koblenz, Mitarbeiter des Rheinischen Kirchenblattes und der Katholischen Blätter. 13,38 sowie $13,220 \mathrm{f}$

Selb, Professor für Theologie in Mainz, seit 1907 mit Becker Herausgeber und Redakteur des Katholik. 1 I, 196.

Seltmann, S. E., Herausgeber und Chefredakteur, Kanonikus in Breslau, 1901 Chefredakteur des Schiesischen Pastoralblattes. KU, 979. - Seit 1879 Herausgeber der Zeitschrift Ut Omnes Unum. 9,71 .

Sickenberger, Joseph, Herausgeber, * 19. 3. 1872 + 27. 3. 1945 Kitzbühel, 1896 Priester, 1903 Professor in München, später in Würzburg und Breslau, 1903-1929 Mitherausgeber der Biblisehen Zeitschrift. L IX, 732, vgl, 9,93 und KK, 1066 . 
Siebold, Pfarrer, Mitglied des Verwaltungsrates der Rheinischen Volkshalle und des literarischen Komitees. 1 lI, 20 und 88. - Mitbegründer der Jahrbiucher des Verelng vom HI. Vincenz von Paul. 1 I, 253.

Smets, Wilhelm, Gründer, Herausgeber, Chefredakteur, Redakteur und Mitarbeiter, ${ }^{\star}$ 15. 2. 1796 Reval, 1822 Priester, 1818 Gründer des Rheinlschen Herold, 1825-27 Chefredakteur der Rheinischen Flora in Aachen, Mitarbeiter der Cölestina, Redakteur der Kölnischen Zeltung, Redakteur des Aachener Album für Leben, Kunst und WIssen, Gründer der Katholischen Monatsschrift zur Belehrung, Erbauung und Unterhaltung in Köln. 13, $104 \mathrm{f}$., vgl, 1 I, 250 sowie 9, 26. - Herausgeber und Redakteur der Katholischen Monatsschrift zur Belehrung, Erbauing und Unterhaltung. 13, 201. - Mitarbeiter der Kathollschen Kirchenzeltung in Aschaffenburg. 13, 203. - Mitarbeiter des Nathanael. 13, 221.

Sprenger, Plazidus, Gründer und Herausgeber, Benediktiner. Mitbegründer des Literarisehen Magazins für Katholiken und deren Freunde 1792 , Herausgeber und gleichfalls auch Gründer der Literatur des Katholischen Deutschlands 1775. 17, 40 , vgl. 9,17 .

Stiss, Johann Joseph, Pfarrer in Godorf bei Köln, Verleger und Herausgeber des Brüderblatt. 1 II, 55.

Schäffer, Sebastian Georg, Herausgeber, *18. 4. 1828 Koblenz, † 9. 11. 1901 Köln, 1852 Priester, Nachfolger Kolpings für die Gesellenvereine, 1891 Domkapitular, Herausgeber der Rheinischen Volksblätter. KK, 4195.

Schäffler, J., Herausgeber und Redakteur, Dr. theol., Pfarrer in Stadtamhof, Redakteur des Volksfreund in Regensburg bis 1877, Herausgeber und Redakteur des Kreuz in Regensburg. 19, 68.

Schanx, Paul (von), Herausgeber, * 4. 3. 1841 Horb in Württemberg, $t$ 1. 6. 1905 Tübingen, 1866 Priesterweihe, 1876 Professor in Tübingen. KK, 4210. - Mitherausgeber der Tübinger Theologischen Quartalschrift.

Schaufert, Geistlicher, Redakteur der Bayrischen Zeitung in München, 1880 Flucht vor der Verhaftung wegen mehrerer „Pressevergehen“. 18, 214.

Scheeben, Matthias Joseph, Herausgeber und Mitarbeiter, 1. 3. 1835 Meckenheim bei Bonn, +21 . 7. 1888 Köln, 1860 Professor für Dogmatik in Köln, 1864-66 Herausgeber des Katholischen Hausfreunds. KK, 4221. - Herausgeber der Periodischen Blatter in Regensburg. 18, 234, vgl. 7 II, 307 sowie 9, 71. - Mitarbeiter des Kathollk. 9, 45. - Mitarbeiter der Kölnischen Volkszeltung. 1 III, 79. - 1862-66 Mitarbeiter des Chilianeum. 9, 44 .

Schelbelberger, Friedrich, Chefredakteur, * 1838 Breitenberg in Bayern, + 21. 6. 1891 Linz, seit 1861 Seelsorger, Chefredakteur des Linzer Volksblattes und der Katholischen Blätter 1876-1880. $\mathrm{KK}, 4230$.

Schelbleln, Georg, Pfarrer in Schwerlenbach, mit Liebermann Redakteur des Katholik 1824-26. 9, 20. vgl. 1 I, 196 .

Schreiner, Josef, Gründer, * 13. 3. 1798 BöhmischLeipa, $t$ 12. 8. 1867 Wien, 1821 Priester, 1824 Professor, 1855 Domherr in Wien, 1850 Mitbegründer der Zeitschrift filr die gesamte katholische Theologie. $\mathrm{KK}, 4236$.
Schiek, Andreas, Gründer, ‘ 17. 8. 1834 Erfurtshausen in Oberhessen, $t$ 1. 10. 1898 Fulda, 1857 Priester, Professor in Fulda, 1884 Grander des Bonifatiusboten 1884. $\mathrm{KK}, 4270$.

Schings, Joseph, + 14. 5. 1876, Kaplan in Aachen, Mitbegründer, Redakteur und Selbstverleger der Christlich-Sozlalen Blătter 1868. 9, 47.

Schlecht, Raimund, Grũnder, * 11. 3. 1811 Eichstātt, + 24. 3. 1891 ebenda, 1834 Priesterweihe, Pädagoge, Mitbegründer der Kathollschen Schulzeitung in Donauwörth. $\mathrm{KK}, \mathbf{4 3 0 0}$.

Schleyer, Johann Martin (Pseudonym Bruder Hilarius Frohsang), Herausgeber, Chefredakteur und Redakteur, '18. 7. 1831 Oberlauda in Baden, + 15. 8. 1912 Konstanz, 1856 Priester, Prălat in Meßkirch, Erfinder einer Weltsprache Volapulk, 1876-86 Chefredakteur der Neuen Sionsharfe. KK, 4311. - Mitherausgeber der Freiburger Zeitschrift für Theologie. 1 I, 244, vgl. 9, 20. Redakteur der Neuen Slonsharle. 18, 670.

Schmeddinck, Benedikt, Grūnder, Herausgeber und Redakteur, Priester der Diözese Münster, um 1848 in Büderich bei Neuf Kaplan, spāter Lehrer in Namur/Belgien, Mitglied des Gründungskomitees der Rheinischen Volkshalle. 13, 52 I., vgl. 1 II, 8. Mitarbeiter des Rheinischen Kirchenblattes. 13,54 u. 219. - Mitarbeiter der KathoIlschen Blätter in Düsseldorf. 13, 221. - Mitarbeiter des Christkatholischen Magazins zur Belehrung und Erbauung fïr alle Stände. 13,214 . Herausgeber und Redakteur des Klemens August oder Katholische Stimme am Rheln. 1. II, $1 \mathrm{f}$

Schmettler, Fr., Kaplan, Redakteur und Mitarbeiter der Westfälischen Volkszeitung in Bochum. 18,623 f., vgl. $9,63 \mathrm{f}$.

Sehmidt, Franziskaner, Expeditus, Herausgeber der literarischen Zeitschrift Uber den Wassern 1908-1915. 9, 93.

Schmidt, J. G., Herausgeber und Chefredakteur Pfarrer in Steinbach bei Lahr, seit 1830 Redak teur der Katholischen Kirchenzeltung in Offenbach. 1 I, 206. - Herausgeber und Redakteur der Katholischen Kirchenzeltung. 13, 202.

Schmitter, Pfarrer in Hennef, 1845-49 Mitarbeiter des Nathanael 13, 107 u. $221 \mathrm{f}$.

Schınittmann, Johann Hermann Joseph, Chefredakteur, " 22. 7. 1807 Krefeld, + 21. 2. 1873 als Pfarrer in Sechtem, 1845 49 Chefredakteur des Nathanael sowie der daran anschließenden Kirchen- und Sehulzeitung. 13, 97 u. 221. - Seit 1849 Redakteur des Nathhanael, Klrchen- und Schulaeltung für das katholische Deutschland. 1 I, 249 , vgl. 1 II, 4

Schmitz, Kaplan aus Trier, Redakteur des Bamberger Volksblattes. $18,224$.

Schmitx, Hermann Joseph, Herausgeber, * 16.5 $1841 \mathrm{Köln},+21$. 8. 1899 ebenda, 1866 Dr. theol und Priester, 1868 Kaplan in Düsseldorf, 1893 Weihbisch of von Köln. A LIV, 128, - Betrieb die Gründung des Augustinus-Vereins. L IX, 436. - Mitherausgeber des Düsseldorfer Sonntagsblattes. 6,25 .

Sehmitz, Johann Hubert, Plarrer, * 1882, Grūnder des Schulfre,undes in Hamm/Westfalen. 9, 26.

Sclımitr, Peter Matthias Hubert, Gründer, Chefredakteur, Redakteur und Mitarbeiter, 1821 Neuf, 1846 Priester, Rektor in Neuß, Chefredakteur des NeuBer Wochenblattes, Redakteur und Mitarbeiter der Christllch-Sozialen Blatter. 6, 53. - Mitbegründer der Neuß-Grevenbroicher Zeltung. 6,53 . 
Schneemann, SJ, Gerhard, Gründer und Chefredakteur, " 12. 2. 1829 Wesel, t 20. 11. 1885 Kerkrade in Holland, 1851 Jesuit, 1858 Professor, Mitbegründer und seit 1879 Chefredakteur der Stimmen aus Maria Laach. L IX, 439, vgl, 18, 53.

Sclneider, SJ, Joseph S., Mitarbeiter, " 5. 9. 1824 Bliesheim, t 7. 1. 1884 Rom. 1850 Priesterweihe, 1852 als Jesuit in der Seelsorge tätig. Mitarbeiter der Stimmen aus Maria-Laach und des Kölner Pastoralblattes sowie anderer Zeitschriften. A XXXII, 132

Schneider, Wilhelm, Нerausgeber, * 4.9.1847 Gerlingen bei Olpe, + 31. 8. 1909 Paderborn. 1872 Priester, 1887 Professor in Paderborn, $1900 \mathrm{Bi}-$ schof daselbst. L IX, 441. - Mitherausgeber des Magazin flír Pädagogik 1879. 18, 99.

Schnütgen, Johann Wilhelm Alexander, Gründer, Herausgeber und Chefredakteur, * 22. 2 . 1843 Steele bei Essen, + 24. 11. 1918 Listernohl. 1866 Priester, 1887 Domkapitular, 1888 Gründer der Zeitschrift für Christliche Kunst. L IX, 443. Erster Herausgeber der Zeitschrift für Christliche Kunst. - Herausgeber und Chefredakteur der Zeitschrift flir Christliche Kunst (1901). KU, 1216.

Schönen, W., Chefredakteur des Gregoriusblattes in Düsseldorf (1901), Pfarrer in Lennep. KU', 458

Schöpf, Joseph Anton, Redakteur, * 5. 2. 1822 Oetztal in Tirol, $t$ 21. 11. 1899 Salzburg, 1845 Priester, 1848 auf Geheiß des Bischofs IRdakteur der Salzburger Constitutionellen Zeitung, 1852 Professor in Salzburg. 1 II, 180 und $190 \mathrm{f}$.

Schwarz, Franz Josef, Gründer und Herausgeber, * 30, 3. 1821 Donzdorf, t 1, 7. 1885 Ellwangen, 1845 Priester, Pfarrer in Böhmen und seit 1868 in Ellwangen, Erneuerer kirchlicher Kunst. I IX, 535. - Seit 1883 Ilerausgeber des Archivs fïr christliche Kunst in Rottenburg. 9, 47 und 73. - 1871 Gründer des Ellwanger Katholischen Kirchenblattes. 19, 43f., vgl, 18, 94. - Mitherausgeber des Stuttgarter Kirchensehmucks. 9, 47.

Schwarz, Wilhelm Eberhard, Herausgeber, Chefredakteur und Redakteur, '20. 4. 1855 Nordkirchen, $t$ 20. 12. 1923, 1878 Priester, Dr. theol., Chefredakteur des Liboriusboten lis 1880, 1898 geschäftsführender Redakteur und 1902-1905 Herausgeber der Germania, später Domkapitular in Münster. 6, 48, vgl. 9, 77 .

Scholz, Johann Martin Augustin, Herausgeber, - 8. 2. 1794 bei Breslau, $t 20$. 10. 1852 Bonn, 1825 Professor für Kirchengeschichte in Bonn. L IX, 449. - Herausgeber der Katholischen Zcitschrlft für Wissenschaft und Kunst. 13, 220 . Mitherausgeber der Zeitsehrift Iïr Philosophie und Katholische Theologie. 1 I, 244, vgl. 9, 20.

Schröder, Josef Wilhelm, Verleger und Redakteur, - 1834, + 25. 5. 1910, Domvikar in Paderborn und mit Rebbert Leiter der Bonifatiusdruckerei, in der der Llboriusbote und das Sonntagsblat Leo verlegt wurden. 1867-96 Redakteur des Bonifatiusblattes. $6,48 f$.

Schröteler, Mitarbeiter, Pfarrer in Bonn-Kessenich, 1846 Oberdirektor der Ritterakademie in Bedburg, Oberpfarrer in Viersen, Mitarbeiter der Katholischen Zeitschrilt fïr Wisserschaft und Kunst 13, 131 und 220. 1857 Priester, Professor in Paderborn, 1890 Domdechant in Paderborn. Mitarbeiter der Frankfurter Zeitung und der Essener Volkszeitung, 6.89.

Schumacher, IHubert, Redakteur, * 1845 Hagen, + 16. 6. 1911 Warendorf, 1870 Priester, 1874-76 Redakteur am Westfälischen Merkur, dann Redakteur des Münchener Sonntagsblattes. $1885 \mathrm{Vi}$ kar, 1900 Missionar in Warendorf. 6, 44

Schwarzenberg, Friedrich Fürst zu, Gründer - 6. 4. 1809 Wien, t 27. 3. 1885 ebenda, 183 priester in Salzburg, 1846 Erzbischof ebenda, 1842 Kardinal. L IX, 536. - Mitbegründer de Salzburger Constitutionellen Zeitung. 1 II, 180 .

Stadlbauer, Max von, Herausgeber und Mitarbeiter, * 13. 7. 1808 Kirchenthumbach in Bayern, † 5. 9. 1866 Rosenheim. 1831 Priester. 1834 Professor in Freiburg, später Rektor der Universilät München. A XXXIV, 378. - Mitherausgeber des Archivs für theologische Literatur. 9. 20, vgl. 1 I, 244. - Mitarbeiter der Neuen Sion. $13,224$.

Stamminger, Johann Baptist, Herausgeber und Chefredakteur, * 6. 3. 1836 Zell a. Main, t 10 12. 1892 Würzburg, 1859 Priester, 1862-66 und 1869 Herausgeber des Chilianeuin, 1877-85 Herausgeber der Katholischen Studien, 1879-84 Redaktenr der Literarischen Rundschau fiir das Katholische Deutschland. A LIV, 440, vgl. 9, 44 und 70 .

Staudenmaie,r, Franz Anton, Herausgeber, Redakteur und Mitarbeiter, * 11. 9. 1800 Donzdorf in Württemberg, +19.1 .1856 Freiburg, 1827 Priester, 1837 Professor in Freiburg und 1843 Domkapitular. L IX, 1024. - 1839-51 Mitherausgeber der Zeitschrift filr Theologie in Freiburg. 19, 28. - Redakteur am Süddeutschen Katholisehen Kirchenblatt. 1 I, 259, vgl. 9, 12. - Mitarbeiter der Süddeutschen Zeitung für Kirche und Staat. 19,28 - Mitarbeiter der Kirchenzeitung für das Katholische Deutschland. 13, 204. - Mitarbeiter des Katholischen Museum. 13, 206. - Mitherausgeber der Jahrbücher für Theologie und Christliche Philosophie. 1 I, 244, vgl. 9, 20.

Stein, Albert Gereon, Mitarbeiter, * 29. 9. 1809 Köln, + 10. 6. 1881 ebentla. 1833 Priester, 1862 Pfarrer, Schriftsteller auf kirchenmusikalischen Gebiet. A XXXV, 505. - Mitarbeiter am Nathanael. 13, 207 und 221 .

Stöveken. Hermann, Redakteur, * 7. 10. 1811 Rheine, 1836 Priester, Kaplan in Köln. 13, $107 \mathrm{f}$ - 1845-49 Redakteur des Nathanael in Köln. 13, 221 , vgl. 1 I, 249 .

Stolle, Franz, Pfarrer und Superior in Leipzig, Herausgcber des Katholisehen Monatsblattes. 19, 49

Stolz, Alban Isidor, Herausgeber und Mitarbeiter, - 3. 2. 1808 Bühl in Baden, + 16.10.1883 Freiburg i. B., 1833 Priester, 1847 Professor in Freibura, bedeutender Volksschriftsteller. L IX, 1003. - Mitarbeiter der Herz-Maria-Blüthen. 19, 79. - Mitarbeiter des Katholischen Hausfreundes. 13, 225. - Herausgeber des Kalender für Zeit und Ewigkeit. 16, 75. - Mitarbeiter des Sïddeutschen Katholischen Kirchenblattes. 13, 213.

Thiel, Andreas, Herausgeber, Redakteur und Mitarbeiter, *28. 9. 1826 Lokau/Ostpreußen, † 17. 7. 1908 Frauenburg, 1849 Priester, 1855 Professor, 1885 Bisch of von Ermland. L X, 112 Mitherausgeber und Redakteur des Schlesisehen 
Kirchenblattes, 13, 208, vgl. 1 I, 246. - Mitarbeiter des Bonner Theologischen Literaturblattes. Aut (1. Jg. 1866, Register).

Thlssen, Eugen, Herausgeber und Mitarbeiter, Stadtpfarrer in Frankfurt, 1852 Preulische Landtagsabgeordneter, Mitarbeiter der Deutschen Volkshalle. 1 II, 297 u. 316 u. 359 . - Heraus geber der Frankfurter Zeitgemäßen Broschüren. 9 44. - Mitarbeiter der Kölnischen Blätter (1866). 6, 14. - Herausgeber des Nassauer Boten in Limburg. 18,590 .

Tinkhausen, G., Professor der Kath.-Theol. Fakultät in Innsbruck um 1843 , ebenda Mitarbeiter der Katholisehen Blätter aus Tirol. 1 I, 249.

Uhl, Stephan, Verleger und Chefredakteur, * 24 12. $1824, t$ 10. 6. 1880 , Priester, Stadtpfarrverweser in ludwigshaven, seit 1857 Chefredakteur, seit 1875 Besitzer des Deutschen Volksblattes in Stuttgart. 6. 14, vgl. 9, 33 und 1 II, 163 .

Vering, Friedrich Ifeinrich, * 9. 3. 1833 Liesborn i. W., $t$ 30. 3. 1896 Prag, 1875 Professor in Czernowitz, später Prag, 1861-96 Herausgeber des Archivs für katholisches Kirchenrecht. L X, 707, vgl. $9,71$.

Vogeisang, V., Gründer, Herausgeber und Mitarbeiter, Theologie-Professor in Bonn, 1832 Mitbegründer der Zeitschrift für Philosophie und Theologie. 9, 20, vgl. 1 I, 244. - Seit 1847 Mitherausgeber der Katholischen Zeitschrift flir Wissenschaft und Kunst. 13, 220. - Mitarbeiter der Katholischen Studien. 9, 74

Vogt, Eduard Franz Anselm, Redakteur und Mit arbeiter, *20. 4. 1814 Ehingen, + 8. 5. 1880 Betzenweiler, 1837 Priester, 1844 Pfarrer in Ludwigsburg, 1877-79 Mitarbeiter des Deutsehen Volksblattes, 1848-49 Redakteur des Kirchlichen Wochenbiattes aus der Dibzese Rottenburg. A $\mathrm{XL}, 178$.

Vosen, Christian Hermann, Redakteur und Mitarbeiter, *9. 7. 1815 Köln, + 12. 5. 1871 ebenda 1844 Religionslehrer in Köln, enger Mitarbeiter von Kolping. L X, 895. - 1839 Priesterweihe, seit 1849 mit Kolping Redakteur des Rheinischen Kirchenblattes. 16, 209, vgl. 1 II, 4, - Redakteur des Christlichen Stadt- und Land boten. 1 II, 55. - Mitarbeiter der Kölnlschen Blätter. 6, 14, vgl. 9, 41. - Mitarbeiter der Rheinischen Volksblätter. 16, 209 - Mitarbeiter des Bonner Theologlschen Literaturblattes. Aut (1. Jg. 1866, Register)

Wachter, Kaplan in Bamberg, (1901) Mitherausgeber des St. Hedwigblattes in Bamberg. KU, 966.

WaIck, Pfarrer in Konzenberg bei Burgau. KU, 575. - Redakteur der Katechetischen Blätter in Dingolfing. 18, 220, vgl. 19, 65 .

Wanner, Gustav, t 1878, Priester, Redakteur des Deutschen Volksblaltes seit 1. 1. 1875. 9, 63.

Wawrezcko, J., Kaplan, Herausgeber und Redakteur des Petrus oder der Fels in der Brandung der Jahrhunderte (1846) in Berlin. 13, 223.

Weber, Beda (Taufname Johanil Chrysanth), Gründer, Redakteur und Mitarbeiter, 28.10 . 1798 Lienz in Tirol, + 18. 2. 1858 Frankfurt, 1820 Benediktiner, 1826 Professor in Meran, $1848 \mathrm{Ab}$ geordneter der Paulskirche in Frankfurt, 1849 Stadtpfarrer in Frankfurt und Domkapitular in Limburg, 1853 Gründer des Frankfurter Katho- lischen Kirchenblattes, 1855 Mitbegründer der Zeitung Deutschland. L $X, 971$. - Mitbegründer des Frankfurter Katholischen Kirchenblattes 1853, 9, 25 u. 46. - Redakteur der Deutschland, 1 II, 442, vgl. 6,18 . Mitarbeiter der Kathollschen Blätter aus Tirol. 1 I, 249 .

Weber, Ingenuin, Herausgeber und Redakteur, 1843 Professor in Innsbruck, Herausgeber und Redakteur der Katholischen Blätter aus Tirol. 1 I, 249.

Weinhart, Benedikt, Mitarbeiter, * 19. 3. 1818 Kempten, + 3. 3. 1901 Freising, 1840 Priester, 1843 Professor in Speyer, später in Freising. L X, 998. - Mitarbeiter der Katholischen Zeitschrift flir Wissenschaft und Kunst. 1 I, 195 . Mitarbeiter des Bonner Theologisehen Literaturblaites. Aut (1. Jg. 1866, Register)

Weis, Nikolaus von, Gründer, * 8. 3. 1796 Schönhof in Lothringen, $t$ 13. 12. 1869 Speyer, 1818 Priester, Professor, 1842 Bischof von Speyer, begründete 1821 zusammen mit RäB den Katholik. I X, 998, vgl. 9, 21 und 2, 79.

Welz, Herausgeber und Redakteur, Erzpriester in Striegau/Schlesien, langjähriger Redakteur des Schiesischen Kirchenblattes, dann Herausgeber und Redakteur des Schlesischen Bonlfatius-Vereinsblattes. 19,121 .

Wengert, Josef, Pfarrer in Dirgenheim/Württenberg, seit 1873 Redakteur des Ipf. 18, 96.

Werber, Friedrich, Chefredakteur, Kaplan in Ra. dolfzell am Bodensee, Redakteur der Freien Stimme rom See. 18,48 , vgI. 19,32 und KÜ, 398.

Werk, Franz Xaver, Ilerausgeber, * 26. 5. 1769 Steinbach bei Bühl, + 26. 12. 1856, 1792 Priester, 1804 Professor in Heidelberg, 1807 in Freiburg. A LV 43. - 1839-51 Mitherausgeber der Zeltschrift für Theologie in Freiburg. 1 I, 244.

Werkmeister, Benedikt Maria, Herausgeber, * 22 . 10. 1745 Füssen, + 16. 7. 1823 Stuttgart, $1765 \mathrm{Be}-$ nediktiner, Professor in Nercsheim und Freising, 1817 Oberkirchenrat. L X, 1054. - llerausgeber der Jahresschrift fir Theologie und Kirchenrecht. 9,19 .

Werner, Zacharias Friedrich Ludwig. Mitarbeiter, 18. 11. 1768 Königsberg, + 17. 1. 1823 Wien 1814 Priesterweibe nach Konversion, Dichter und Dramatiker. L X, 1056. - Mitarbeiter des Katholischen Museums. 13, 205. - Mitarbeiter der Cölestina. 1 I, 280, vgl. 9, 27.

Werthmann, Lorenz, Gründer, * 1. 10. 1858 Geisenheim, +10, 4. 1921 Freiburg, 1883 Priester Bischöfl. Kaplan in Limburg und Freiburg, Gründer und Präsident des Deutschen Caritasverbandes. L X, 1060. - Gründer der Zeitschrift CarItas. 9,94 .

Wessenberg, Ignaz Heinrich Frhr. von, lIerausgeber und Mitarbeiter, * 4. 11. 1774 Dresden, 9. 8. 1860 Konstanz, 1798 Kanonikus in Konstanz, 1802 Generalvikar, 1802-1804 Herausgeber der Geistlichen Monatsschrlft und des Archiv für $\mathrm{Pa}$ storalkonferenzen, Vertreter beim Wiener KongreB. L X, 1064. - Herausgeber des Archlv filr Pastoralkonferenzen 1802-27. 1I, 245, - Mitarbeiter des Katholischen Museums. 13, 206. - Mitarbeiter des Christkatholischen Religionsmagazins. 13,206 
Wesermayer, Anton, Gründer, Herausgeber und Redakteur, Mitarbeiter, * 2. 1. 1816 Deggendorf, † 3. 12. 1884 München, Stadtpfarrer in München, Mitglied des Deutschen Reichstages und bayrischer Landtagsabgeordneter, Herausgeber des Kathollschen Hausfreundes, Schriftsteller. A XLII, 186. - Gründer und 1851-56 auch Herausgeber des Katholischen Hausfreundes, 9, 48. - Herausgeber des Katholischen Hausfreundes 1846-56. 1 I, 250, vgl. 9, 27. - Herausgeber des Katholischen Volksire,und in München 1 II, 174. - Herausgeber und ab 1845 auch Redakteur der Passauer Katholischen Klrchenzeitung. 13, 215. Redakteur und Herausgeber des Kathollschen Hausfreundes. 13, 225.

Wichert, Johann, Redakteur, " 1850 Tolksdorf bei Braunsberg, 1874 Priester, 1877 Redakteur der Ermländer Zeltung, 1905 Generalvikar, 1916 Domdekan in Braunsberg. 6, 95.

Wick, Joseph Lorenz, Grunnder, * 27. 8. 1820 Mellenthin in Westpreußen, t 9. 2. 1903 Breslau, 1845 Priester, 1872 Domkapitular in Breslau und später Domprediger ebenda. L X, 1091. - 1863 Gründer der Breslauer Hausblätter für das Volk. 6, 21, vgl. 7 II, 302. - Gründer der Schlesischen Volkszeifung. 9, 59

Wleser, Sebastian (Ps. Ausshart), Mitarbeiter, " 15. 1. 1879 Kirchweihdach in Oberbayern, Pfarrer und Schriftsteller in Augsburg. KL, 3369. Mitarbeiter der Deutsehen Katholikenzeltung in München seit 1920. 9, 94

Wintersteln, Dr., Domprediger in Würzburg, 1901 Chefredakteur des Würzburger Katholischen Sonntagshlattes. KU, 1200

Wiser, Thomas, Herausgeber und Redakteur, - 15. 4. 1810 Straubing, t 6. 8. 1879 Regensburg, 1835 Priesterweihe, 1868 Kanonikus in Regensburg und Dekan. 1845-49 Redakteur der Slon. A XXXXIII, 538. - Seit 1844 Herausgeber, 184447 allein und 1847-48 zusammen mit Reithmayr Redakteur der Slon. 13, 204, vgl. 9, 23.

Witt, Franz Xaver, Gründer und Herausgeber, - 9. 2. 1834 Walderbach, + 2. 12. 1888 Landshut, 1856 Priester, 1867 Gründer des Allgemeinen
Deutschen Cäcilienverbandes. L X, 1197. - Gründer und Herausgeber der Fliegenden Blätter für Katholische Kirchenmusik 1866 in Regensburs und 1868 der Musica Sacra ebenda. 9, 74 und 47, vgl. $\mathrm{KU}, 765$

Woker, Dr., Chefredakteur, Domkapitular in Paderborn, 1901 Chefredakteur des Bonifatius. blattes in Paderborn. KU, 174. - Chefredakteur des Katholischen Seelsorgers. KU, 778.

Wurm, Alois, Gründer, Herausgeber und Mitarbeiter, * 20. 12. 1874 Straubing, Dr. theol., Schriftsteller in München, Gründer und Herausgeber der Zeitschrift Seele. KL, 3475, vgl. 9 94. - Mitarbeiter der Alten und Neuen Welt Aut (35. Jg. 1901, S. 614-18).

Zarbl, Johann Baptist, Herausgeber und Mitarbeiter, * 7. 6. 1794 Eggerding im Innertal, $t 30$ 6. 1862, 1819 Priester, 1830 Professor in Freising, 1848 Domprobst in München, Mitarbeiter der Eos 1829-30. A XXXXIV, 695. - Herausgeber des Seelsorger 1839-45. 9, 20.

Zeck, Karl Wilhelm, Redakteur und Mitarbeiter, * 17, 8. 1852 Rheinberg + 14, 9. 1910, 1883 Priester, 1901 Pfarrer in Ulpenich. 6, 46. - Redak teur des Düsseldorfer Sonntagsblattes und Mitarbeiter der Wuppertaler Volksblätter bis 1886 6,46 und 50 .

Zetter, Max Theophil, Pfarrer, seit 1851 Redakteur der Salzburger Constitutionellen Zeitung. 1 II, 186 .

Zingerle, Jakob Ordensname Pius, * 17. 3. 1801 Meran, $t 10.1$. 1881 Marienburg in Südtirol 1824 Priester, spâter Lehrer und Lektor im Stift Marienburg. L X, 1373. - Mitarbeiter der Katholischen Blätter aus Tirol. 1 I. 249. - Mitarbeiter am Hausbuch für Christliche, Unterhaltung. 9, 48.

Zirkel, Gregor, Gründer, * 2. 8. 1762 Sylbach bei Haßfurt, t 18. 12. 1817 Würzburg, 1786 Priester, 1797 Professor in Würzburg, 1802 Weihbischof, 1817 Bischof von Speyer. L X, 1381. Maßgeblich an der Gründung der Literaturzeitung für katholische Religlonslehrer beteiligt. 1 J, 183.

\section{E N D E}




\section{Schlüssel der Fundstellen-Signierungen}

\section{in den „Fachlich-biograpbischen Notizen" (Beilage)}

Nr. oder Buchstabe

Titel

A Allgemeine Deutsche Biographie, Leipzig 1875-1912.

KK Wilhelm Kosch, Das katholische Deutschland, 3 Bde., Augsburg 1933-38.

KL Wilhelm Kosch, Deutsches Literaturlexikon, 4 Bde., 2. Auflage Bern 1949-58.

KƯ Joseph Kürschner, Handbuch der Presse, Berlin-Eisenach-Leipzig 1902.

L Lexikon für Theologie und Kirche, 2. Auflage Freiburg 1957-65.

N Neue Deutsche Biographie, Berlin $1953 \mathrm{ff}$.

1 Karl Bachem, Josef Bachem und die Entwicklung der katholischen Presse in Deutschland, Köln, Bd. I 1912, Bd. II 1913, Bd. III 1938.

2 Hermann Baumhauer, Die Anfänge der katholischen Presse im Elsaß, Konstanz 1939.

3 Ludwig Bergsträsser, Politischer Katholizismus, Dokumente seiner Entwicklung: München 1923.

4 Ludwig Bergsträsser, Studien zur Vorgeschichte der Zentrumspartei, Beiträge zur Parteiengeschichte, Tübingen 1910.

5 W. Hubert Ganser, Die Süddeutsche Zeitung für Kirche und Staat Freiburg 1845-48. Eine Studie über die Anfänge des politischen Katholizismus in Baden, Berlin 1936.

6 Wilhelm Kisky, 50 Jahre Augustinus-Verein zur Pflege der katholischen Presse, Düsseldorf 1928.

7 Johannes B. Kissling, Geschichte des Kulturkampfes im Deutschen Reich, Freiburg, Bd. I 1911, Bd. II 1913, Bd. III 1916.

8 Kurt Koszyk, Deutsche Presse im 19. Jahrhundert, Berlin 1966.

9 Klemens Löffler, Geschichte der katholischen Presse Deutschlands, M.-Gladbach 1924.

10 Paul Majunke, Geschichte des Kulturkampfes, Freiburg 1911-13.

11 J. W. Naumann, Die Presse und der Katholik, Anklage und Rechtfertigung, Augsburg 1932.

12 [Andreas Niedermayer], Die katholische Presse Deutschlands, Freiburg 1861.

13 Rudolf Pesch, Die kirchlich-politische Presse der Katholiken in der Rheinprovinz vor 1848, Mainz 1966.

14 Hans Joachim Reiber, Die katholische deutsche Tagespresse unter dem Einfluß des Kulturkampfes, Görlitz 1930.

15 Alfons Runte, Das soziale Schrifttum Bischof Kettelers, Diss. Münster 1935.

16 Michael Schmolke, Adolph Kolping als Publizist, Münster 1966.

17 Franz Wetzel, Geschichte der katholischen Presse Deutschlands im 18. Jahrhundert, Diss. Heidelberg, Mannheim 1913.

18 Leo Woerl, Publizistik der Gegenwart, Eine Rundschau über die gesamte Presse der Welt, Würzburg $1879 \mathrm{f}$.

19 Leo Woerl, Weltrundschau über die katholische Presse, Würzburg 1878.

Aut. Angaben wurden durch Autopsie gewonnen. 